\title{
Flexofytol, a Purified Curcumin Extract, in Fibromyalgia and Gout: A Retrospective Study ${ }^{*}$
}

\author{
Thierry Appelboom ${ }^{1}$, Christian Mélot MsciBiost $^{2}$ \\ ${ }^{1}$ Rheumatology Department, Erasme University Hospital, Medical School, University of Brussels, Brussels, Belgium; ${ }^{2}$ Emergency \\ Department, Erasme University Hospital, Medical School, University of Brussels, Brussels, Belgium. \\ Email: thierry.appelboom@erasme.ulb.ac.be
}

Received February 26 ${ }^{\text {th }}, 2013$; revised March 28 ${ }^{\text {th }}, 2013$; accepted April $9^{\text {th }}, 2013$

Copyright (C) 2013 Thierry Appelboom, Christian Mélot MsciBiost. This is an open access article distributed under the Creative Commons Attribution License, which permits unrestricted use, distribution, and reproduction in any medium, provided the original work is properly cited.

\begin{abstract}
Flexofytol is a recently-purified curcumin extract administered as a food supplement with the indication for being "able to improve musculoskeletal flexibility and preserve articular function”. It is now marketed in Belgium, frequently used with success in daily clinical practice according to pharmacists, and also prescribed by general practitioners and rheumatologists for various painful conditions. The objective of this retrospective study was to evaluate the potential efficacy of the compound in fibromyalgia and gout from our practice from 2011-2012. Accordingly, the medical files of 116 patients (83 with a follow-up of 1 to 12 months) with fibromyalgia or gout who were treated with Flexofytol were analysed to determine whether they had benefited from this therapy or had experienced undesirable effects. Flexofytol provided a benefit in acute gout crisis and fibromyalgia. Interestingly, the clinical improvement was rapid (within 24 $48 \mathrm{~h}$ ), with good efficacy in gout. The benefit of Flexofytol was on different manifestations of pain rather than on the sleep disorders of fibromyalgia. Except for some occasional difficulties with tablet swallowing and transient diarrhoea, the treatment was well tolerated. In conclusion, Flexofytol appears to be an alternative to conventional treatment for fibromyalgia and gout.
\end{abstract}

Keywords: Curcumin; Fibromyalgia; Gout

\section{Introduction}

Flexofytol is a recently purified curcumin extract licensed and marketed by Tilman Laboratory (Belgium). Essentially, it contains diferuloylmethane and 2 derived compounds, demethoxycurcumin and bismethoxycurcumin. In order to facilitate intestinal absorption, the tablet $(42 \mathrm{mg})$ is supplemented with polysorbate as an emulsifier, and citric acid as a pH stabilizer. Consequently, the solubility of Flexofytol is 7500 times greater than that of Curcuma.

The toxicity of Curcuma remains unknown, but dosages of $12 \mathrm{~g}$ daily have been administered for more than 3 months without side effects. A systematic review of curcumin toxicity performed in 2003 concluded that there was an absence of toxicity for dosages up to $8 \mathrm{~g}$ daily, and that the compound has an anti-inflammatory effect. On the other hand, Curcuma claims to exert protective effects on

${ }^{*}$ Conflict of Interest: The authors received a financial support from Tilman Laboratory (Belgium) for publishing their retrospective experience, the statistical analysis and the publication of the data. the gastric mucosa, and to probably have a cicatrizing effect on gastric ulcers (European Commission for Health). In traditional therapy, Curcuma is used in the treatment of rheumatic pain and other clinical conditions including Cancer, Helicobacter pylori infection, scabies [1-4].

The ancient curry spice known as turmeric and its bioactive component, curcumin have been revered in the world of Ayurvedic medicine for more than 2500 years. Curcuma is thought to prevent digestive cancers, improve measures of cognition and brain health, including Parkinson's and Alzheimer's, and to be a maintenance therapy in ulcerative colitis [5,6].

Flexofytol has been marketed in Belgium since 2010 as a food additive for joint protection and improvement of flexibility. The high number of Flexofytol tablets sold in Belgium reflects a high degree of satisfaction among patients. Now, Flexofytol is also prescribed by physicians in daily practice, and improvement in diffuse pain, lower back pain, tendonitis, and arthritis associated with 
Flexofytol is frequently reported. For these reasons, we performed a retrospective analysis of our own experiences, and assessed the tolerance of the compound from our medical files of patients with fibromyalgia and gout.

\section{Patients and Methods}

Our population consisted of 116 patients treated during the end of 2011 and the beginning of 2012 with Flexofytol 4 tablets daily for fibromyalgia $(n=62)$ and gout (n $=54$ ), with a follow up visit after a period of $4-6$ weeks for 83 (42\%) patients. The following information was recorded from the medical files: age, sex, and global assessment of possible benefit. In fibromyalgia, additional information generally available included the following questions: can you rate between 0 (no) and 10 (full) the severity of your symptoms (diffuse pain, fatigue, morning fatigue, abnormal fatigability, headache, dizziness, thoracic oppression, muscle cramps, paraesthesia in the limbs, intolerance to contact, noise, and odours), and the tender points count. The presence of side effects was also recorded.

\section{Statistical Analysis}

The data were presented as a histogram and as medians with interquartile ranges (IQR) before and after treatment. The medians were compared using a nonparametric test for repeated measures (Wilcoxon signed rank test). The percentage of responses before and after treatment was compared using z-test. A p-value $<0.05$ was considered significant.

\section{Results}

\subsection{Effect of Flexofytol in Fibromyalgia}

Among the 62 patients treated with Flexofytol 4 tablets daily, 41 felt that the therapy was beneficial. In the clinical setting of fibromyalgia, diffuse pain and fatigue are central. Therefore, it seemed interesting to look for an effect on these symptoms in particular. Indeed, Flexofytol reduced diffuse pain intensity, with the median (IQR) shifting from $8(7-8)$ to $6(5-8)(p=0.003)$ (Figure 1). A benefit was also observed on fatigue; the median (IQR) indicates a reduction from 8 (6 - 8) to 7 (5 - 8) $(p=0.0392)$ (Figure 2). Concerning morning fatigue, which is often reported by these patients, the difference was not significant $(p=0.0625)$ despite a reduction in the median from 8 (7 - 8) to 6.5 (3.75 - 9). On the other hand, fatigue during domestic work improved with a reduction in median (IQR) from 8 (5 - 9) to 7.5 (5.25 - 8) (p = 0.0143).

Among the other symptoms of fibromyalgia, an important reduction in dizziness was noted, with a median (IQR) reduction from $5(2-8)$ to $3(1-7)(p=0.0258)$, as well as in thoracic oppression with a median decrease

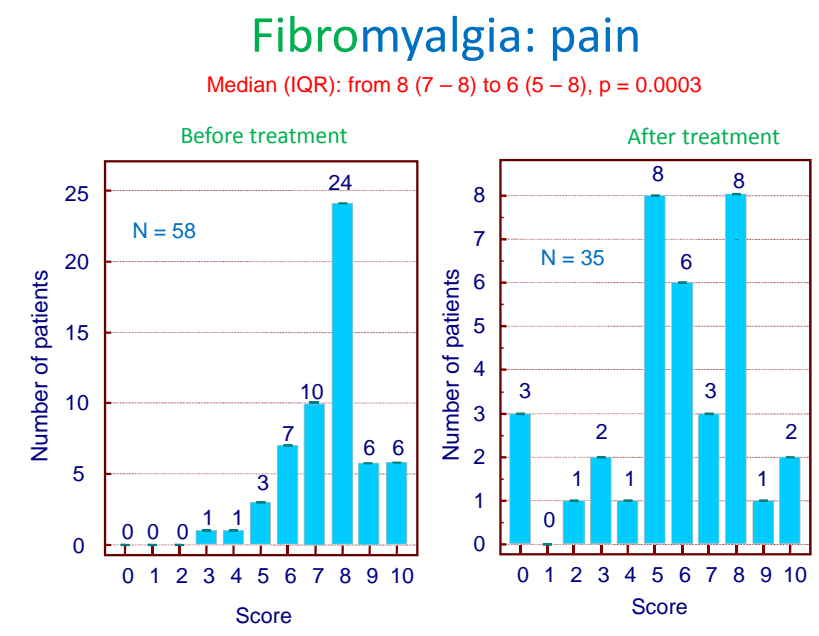

Figure 1. Fibromyalgia: effect of Flexofytol on pain ( $p=$ 0.0003).

\section{Fibromyalgia: fatigue}

Median (IQR): from $8(6-8)$ to $7(5-8), p=0.0392$
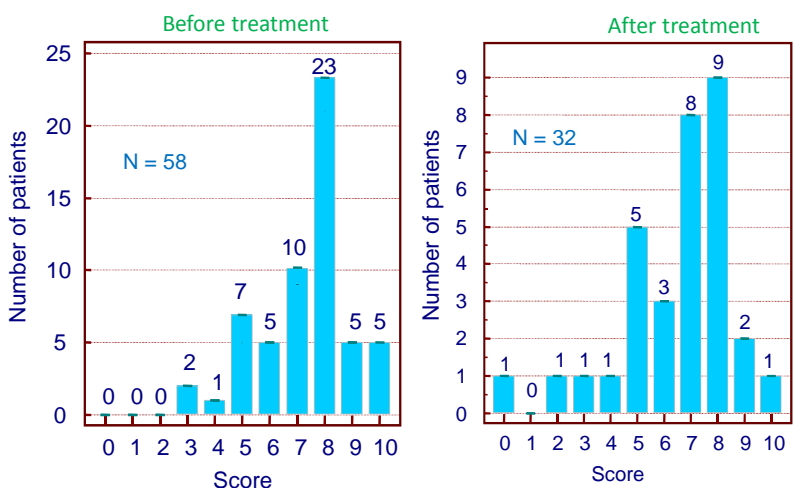

Figure 2. Fibromyalgia: effect of Flexofytol on fatigue ( $p=$ 0.0392).

from $7(6-8)$ to $5(2.5-7)(\mathrm{p}=0.0015)$, heart palpitations with a median reduction from $6(3.75-8)$ to $5(2-6)$ $(\mathrm{p}=0.004)$, cramps from $6(4-8)$ to $4.5(2-7)(\mathrm{p}=$ $0.0096)$, and paraesthesia in the extremities from 7 (4 - 8) to $4(1-7)(p=0.0018)$.

On the other hand, no significant benefit was observed for headache, which remained unchanged from 6.5 (3 - 8) to $6.5(2-8)(p=0.1477)$, perception of painful skin contact from $8(6-8)$ to $7(5-8)(p=0.3343)$, intolerance to noise from $7(4.75-8.25)$ to $7.5(5-8)(p=0.4412)$, or to odours from $6(0-8)$ to $5(0-7)(p=0.025)$. Tender points, which are cardinal for the diagnosis of fibromyalgia and represent diagnostic criteria, were not modified by treatment, with medians (IQR) ranging from 18 (18 $18)$ to $18(8-18)(\mathrm{p}=0.1814)$.

\subsection{Effect of Flexofytol in Gout}

With Flexofytol, 17/19 gout patients reported dramatic 
improvements in acute gout symptoms within $24-48 \mathrm{~h}$ with 4-6 Flexofytol tablets daily $(p=0.0002)$ (Figure 3). Eleven patients decided to spontaneously continue therapy to prevent recurrence by taking 2 tablets daily for 3 - 6 months, and 10/11 were satisfied after 6 months.

\subsection{Undesirable Effects}

Some patients reported difficulties swallowing the tablets ( $n=4 / 83$ ), slight diarrhoea $(n=5 / 83)$, and skin itchiness ( $n=1 / 83$ ) that spontaneously resolved when the dosage was reduced from 4 to 2 tablets daily. When blood testing could be obtained (51/83), no alteration in renal, liver, or hematopoietic function was noted.

\subsection{Drug Interaction}

Our experience is limited, but so far no drug interaction was reported with warfarin $(n=2)$ or antidiabetic agents $(n=4)$.

\section{Discussion}

The most benefit seen with Flexofytol was observed in gout, with a majority of patients (17/19) reporting that they experienced clinical improvement within 24 - $48 \mathrm{~h}$, which was similar to that obtained with non-steroidal anti-inflammatory drugs (NSAIDs). It is important to note that some patients decided to take 6 tablets daily rather than 4 because they considered that a benefit was present, but insufficient with the regular dose. Since this first experience, 20/23 additional patients (not included in this study) reported a benefit from Flexofytol, and confirmed excellent pain relief within 1 or 2 days. The same group decided spontaneously to adopt Flexofytol as maintenance therapy because they felt that it provided a protective effect against the recurrence of gout crisis (data not shown).

In patients with fibromyalgia, a high proportion of pa-

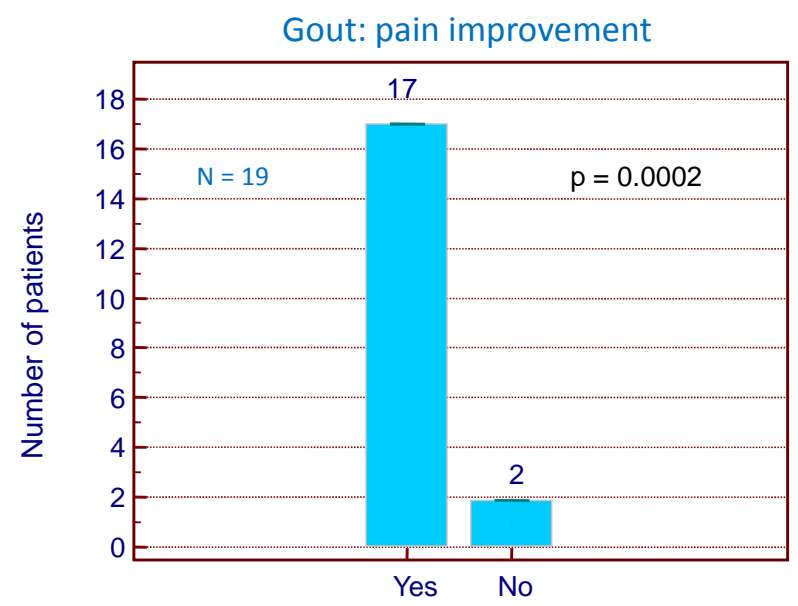

Figure 3. Gout: number of patients improved $(p=\mathbf{0 . 0 0 0 2})$. tients reported experiencing clinical improvement. Interestingly, some symptoms were more improved than others. In decreasing order, paraesthesia, diffuse pain, dizziness, muscle cramps, thoracic oppression, palpitations, and intolerance to odours were improved. Fatigue and abnormal fatigability for domestic work were not improved by Flexofytol, and no benefit was observed for headache, intolerance to contact or noise, or the number of tender points.

Flexofytol was well tolerated. Two patients complained that the relatively large size of the tablet made swallowing difficult, and 2 reported some laxative effects when the dose was increased to 6 tablets daily for more than 10 days.

This retrospective observational study provides only a limited level of evidence. Accordingly, no definitive conclusion can be drawn, but these encouraging results suggest further research and development of curcumin not only as a food additive, but as a new drug. The main advantage of curcumin is its extensive use as a therapeutic agent and as a cooking additive. No adverse events are reported in the literature, even for high dosages. Consequently, at first glance the compound could be indicated for older, polymedicated, anticoagulated, or frail patients, or in those with hepatic or renal alterations.

The good results in gout patients can be explained by the particular mode of action of curcumin. In vitro, curcumin can block production of IL-12 by CD4, CD8, and NK cells, and, consequently, by the production of gamma interferon [7] In addition, curcumin blocks the expression of IL-6 and IL-8 by chondrocytes [8]; it can also stimulate the production of IL-4 and, consequently, inhibit the NF-kappa-B pathway implicated in the production of cytokines.

Other in vitro studies have suggested that the compound could inhibit enzymes involved in inflammation such as phospholipase, cycloxygenase-2, and lipoxygenase, NO production induced by interleukin-1 beta [8,9] and the activity of enzymes like collagenase, elastase, and hyaluronidase [10]; and to trap free radicals, superoxide anions, and some pro-inflammatory metals such as iron and copper [11] Curcumin regulates transcription factors, kinases, adhesion molecules, redox status, and other enzymes involved in inflammation [12].

These in vitro effects are relevant if curcumin can penetrate the intestinal barrier and arrive at the site of inflammation. Penetration of curcumin is highly variable (5\% - 60\%) from one person to another. The main advantage of Flexofytol is its physical transformation into micellae and its chemical design for penetration. This should allow it to achieve high concentrations in the circulation and in peripheral tissues. This penetration characteristic differentiates Flexofytol from its competitors, which are supplemented by piperidine (a pepper extract) or cyclo- 
dextrin, which represent alternatives for facilitating the penetration of curcuma.

Other studies have also suggested an anti-inflammatory role for curcumin since it reduces morning stiffness in rheumatoid arthritis with an effect comparable to NSAIDs. It could also induce partial relief from postoperative pain.

A positive effect was also reported in fibromyalgia patients. An important proportion (42\%) of these patients was lost to follow up, and whether they were satisfied or dissatisfied with the treatment is unknown. If a placebo effect is likely, it is balanced by the high number of fibromyalgia patients who are depressed, rarely express satisfaction with therapy, or do not comply with the treatment plan.

The mode of action of Flexofytol in fibromyalgia is difficult to explain. Interestingly, improvement was noted in decreasing order for paraesthesia, diffuse pain, dizziness, cramps, thoracic oppression, palpitations, and less significantly for fatigue during effort. No effect on headache, intolerance to contact, noise, or on the number of tender points was recorded.

Under these conditions, Flexofytol would play a role in reducing pain rather than affecting sleep. As a hypothesis, the target of Flexofytol could be the grey nuclei of the brain, which play a more central role in pain control than the limbic system, which is active in sleep control.

In conclusion, in this retrospective, real-life study in patients with gout and fibromyalgia, Flexofytol provided a benefit without adverse effects. These results need to be confirmed, and double-blind trials are necessary prior to drawing any definitive conclusion. Yet, if confirmed, Flexofytol could represent a safe and efficacious alternative treatment in these conditions.

\section{REFERENCES}

[1] C. Koosiriatt, S Linpisarn, D. Changsom, K. Chawansuntati and J. Wipasa, "Investigation of the Anti-Inflammatory Effect of Curcuma in Helicobacter Pylori Infected Patients,” International Immunopharmacology, Vol. 10, No. 7, 2010, pp. 815-817. doi:10.1016/j.intimp.2010.04.021

[2] B. Chandran and A. Goel, "A Randomized Pilot Study to Assess the Efficacy and the Safety of Curcumin in Patients with Active Rheumatoid Arthritis,” Phytotherapy Research, Vol. 26, No. 11, 2012, pp. 1719-1725.

\section{doi:10.1002/ptr.4639}

[3] A. S. Strimpakos and R. A. Sharma, "Curcumin: Preventive and Therapeutic Properties in Laboratory Studies and Clinical Trials,” Antioxidants \& Redox Signaling, Vol. 10, No. 3, 2008, pp. 511-545. doi:10.1089/ars.2007.1769

[4] T. Kawamori, R. Lubet, V. Steele, et al., "Chemopreventive Effect of Curcumin a Naturally Occurring AntiInflammatory Agent during the Promotion/Progression Stages of Colon Cancer," Cancer Research, Vol. 59, No. 3, 1999, pp. 597-601.

[5] T. Jida, K. Takeuchi, F. Watanabe, et al., "Curcumin Maintenance Therapy for Ulcerative Colitis: Randomized, Multicenter, Double-Blind, Placebo-Controlled Trial,” Clinical Gastroenterology and Hepatology, Vol. 4, No. 2, 2006, pp. 1502-1506. doi:10.1016/j.cgh.2006.08.008

[6] T. Hamaguchi, K. Ono and M. Yamada, "Review: Curcumin and Alzheimer's Disease," CNS and Neurosciences and Therapeutics, Vol. 16, No. 5, 2010, pp. 285-297. doi:10.1111/j.1755-5949.2010.00147.x

[7] B. Y. Kang, Y. J. Song, K. M. Kim, Y. K. Choe, S. Y. Hwang and T. S. Kim, "Curcumin Inhibits Th1 Cytokine Profile in CD4+ T Cells by Suppressing IL-12 Production in Macrophages,” British Journal of Pharmacology, Vol. 128, No. 2, 1999, pp. 380-384. doi:10.1038/sj.bjp.0702803

[8] M. Mathy, I. Jacquemond-Collet, F. Priem, C. Sanchez, C. Lambert and Y. Henrotin, "Curcumin Inhibits Proinflammatory Mediators and Metalloproteinase-3 Production by Chondrocytes,” Inflammation Research, Vol. 58, No. 12, 2009, pp. 899-908. doi:10.1007/s00011-009-0063-1

[9] V. P. Menon and A. R. Sudheer, "Antioxidant and AntiInflammatory Properties of Curcumin," Advances in Experimental Medicine and Biology, Vol. 595, 2007, pp. 105-125. doi:10.1007/978-0-387-46401-5_3

[10] N. Chainani-Wu, "Safety and Anti-Inflammatory Activity of Curcumin a Component of Tumerin (Curcuma longa)," The Journal of Alternative and Complementary Medicine, 2003 Vol. 9, No. 1, 2003, pp. 161-168. doi:10.1089/107555303321223035

[11] H. Hatcher, R. Planalp, J. Cho, F. Torti and S. V. Torti, "Curcumin: From Ancient Medicine to Current Clinical Trials,” Cellular and Molecular Life Sciences, Vol. 65, No. 11, 2008, pp. 1631-1652. doi:10.1007/s00018-008-7452-4

[12] B. B. Aggarwal and K. B. Harikumar, "Potential Therapeutic Effects of Curcumin, the Anti-Inflammatory Agent, against Neurodegenerative, Cardiovascular, Pulmonary, Metabolic, Autoimmune and Neoplastic Diseases,” The International Journal of Biochemistry \& Cell Biology, Vol. 41, No. 1, 2009, pp. 40-59. doi:10.1016/j.biocel.2008.06.010 